\title{
Introduction of thoracoscopic surgery for congenital pulmonary airway malformation in infants: review of 13 consecutive surgical cases
}

\author{
Atsushi Ito ${ }^{1}$, Motoshi Takao ${ }^{1}$, Akira Shimamoto ${ }^{1}$, Shinji Kaneda ${ }^{1}$, Kohei Matsushita ${ }^{2}$, Mikihiro Inoue ${ }^{2}$, \\ Keiichi Uchida ${ }^{2}$ \\ ${ }^{1}$ Department of Thoracic and Cardiovascular Surgery, ${ }^{2}$ Department of Gastrointestinal and Pediatric Surgery, Mie University School of Medicine, \\ Tsu, Japan \\ Contributions: (I) Conception and design: A Ito, M Takao; (II) Administrative support: M Takao, A Shimamoto, S Kaneda; (III) Provision of study \\ materials or patients: K Matsushita, M Inoue, K Uchida; (IV) Collection and assembly of data: A Ito, K Matsushita, M Inoue, K Uchida; (V) Data \\ analysis and interpretation: A Ito, M Takao; (VI) Manuscript writing: All authors; (VII) Final approval of manuscript: All authors. \\ Correspondence to: Atsushi Ito, MD. Department of Thoracic and Cardiovascular Surgery, Mie University School of Medicine, 2-174 Edobashi, Tsu, \\ Mie 514-8507, Japan. Email: i-atsushi@clin.medic.mie-u.ac.jp.
}

Background: Although complete video-assisted thoracic surgery (C-VATS) has been demonstrated to have several advantages compared with conventional thoracotomy, there are few reports on the clinical feasibility of C-VATS for CPAM in infants.

Methods: We retrospectively evaluated 13 consecutive patients (neonates 4; infants 9) surgically treated for congenital pulmonary airway malformation (CPAM) from 1 January 2008 to 31 March 2017.

Results: In the group of neonates, all 4 cases were prenatally diagnosed and they underwent semi-emergent surgery after birth due to respiratory failure. In the group of 9 infants, 5 cases were prenatally diagnosed and 4 cases were diagnosed at age $>2.5$ years due to symptoms associated with pulmonary cystic infection. Pulmonary resection consisted of the following: 8 lobectomies, 1 segmentectomy, 2 wedge resection, 1 fractionated lung resection and 1 lobectomy with segmentectomy. Overall, there were 9 thoracotomy and 4 thoracoscopic surgeries. Mean operation time was $162 \mathrm{~min}$ (range, 67-290 min) and blood loss was $21 \mathrm{~mL}$ (range, 0-74 mL) on average. There were no complications such as thoracic deformity or respiratory failure, however in 2 of those who underwent segmentectomy the cystic remnant remained. No statistically significant differences were observed between the thoracotomy Group and C-VATS group in terms of age and height at intervention, operation time, blood loss, postoperative day of drain removal, and length of hospital stay after surgery. However, only the average body weight was heavier in C-VATS group ( $\mathrm{P}=0.03)$.

Conclusions: Since early surgical resection of asymptomatic CPAM is often recommended for the prevention of infections and the development of lung malignancy, we recommend performing surgery after the age of 1 year if the patient's condition is stable. Furthermore, C-VATS lobectomy may be feasible if they are older than 18 months or weigh more than $10 \mathrm{~kg}$.

Keywords: Congenital pulmonary airway malformation (CPAM); surgical management; thoracoscopic surgery; infant; neonate

Submitted May 26, 2019. Accepted for publication Nov 19, 2019.

doi: $10.21037 /$ jtd.2019.12.14

View this article at: http://dx.doi.org/10.21037/jtd.2019.12.14 


\section{Introduction}

Congenital pulmonary airway malformation (CPAM) is a disease in which bronchioles abnormally proliferate and form various sizes of cysts in the fetal period. Traditionally, CPAM has been classified according to Stocker (1) into five major types (Type 0 to Type 4 ) based on the histopathological cyst diameter and location. The type of CPAM reflects the progression of developmental abnormalities through the tracheobronchial tree, from large airways to bronchioles and alveoli (2). Although the precise etiology of CPAM is not known, it is thought to be the result of hamartomatous malformation and abnormal proliferation of the pulmonary tissue at different sites (3).

Surgical resection is the standard care for symptomatic CPAM; however, the management of asymptomatic CPAM remains controversial. Although the morbidity and mortality of surgery should be taken into account, many series recommend early surgical resection to avoid the eventual development of complications such as recurrent infections, spontaneous pneumothorax, or even the rare development of lung malignancy (4).

The aim of this study is to report our surgical outcome of CPAM in neonates and infants and to evaluate the optimal timing of surgical intervention including C-VATS.

\section{Methods}

We retrospectively evaluated consecutive patients treated surgically for CPAM in neonates and infants at Mie University Hospital from 1 January 2008 to 31 March 2017. Medical and operative records were retrieved for data analysis. Patient's demographic data included sex, age and weight at surgery, date of diagnosis, clinical history, malformation site, type of surgical resection, pathology examinations using Stocker's classification (1). Surgical outcomes including operative time, postoperative complications and length of hospital stay were also extracted and analyzed statistically. Postoperative complications were defined as having a persistent air-leak ( $\geq 72 \mathrm{hr}$ ), pleural effusion, chylothorax, deep wound dehiscence, blood transfusion, renal failure, or respiratory failure related to the surgery. In general, computed tomography (CT) scan of CPAM shows multi-septated air-filled cystic lesions of segmental lung tissue with abnormal bronchial proliferation.

Patient backgrounds and operation data were compared in infants between the thoracotomy Group and complete video-assisted thoracic surgery (C-VATS) Group. Statistical analysis was performed using Mann-Whitney $U$ test for continuous variables (age and height/weight at intervention, operative time, blood loss, postoperative day of drain removal, and length of hospital stay after surgery). All statistical analyses were performed with EZR (Saitama Medical Center, Jichi Medical University, Saitama, Japan). More precisely, EZR is a modified version of R commander (version 1.6-3) designed to add statistical functions used frequently used in biostatistics. The statistical significance was determined at $\mathrm{P}<0.05$. The study was approved by the Research Ethics Board of the Mie University School of Medicine.

\section{Results}

\section{Characteristics of patients}

Between January 2008 and March 2017, a total of 13 consecutive pulmonary resections were performed for 4 neonates ( 3 boys and 1 girl) and 9 infants ( 3 boys and 6 girls) with CPAM. In the group of neonates, all 4 cases were prenatally diagnosed and they underwent semi-emergent surgery after birth due to respiratory failure; 2 required preoperative ventilator management. Mean age and weight for surgery was 5 days (median 5.5: range 1-8 days) and $2.9 \mathrm{~kg}$ (median 2.85: range 2.2-3.6 kg). The locations of malformation were: 1 right upper lobe, 1 right middle lobe, 1 right lower lobe, 1 left lower lobe.

In the group of infants, 5 cases were prenatally diagnosed and 4 cases were initially diagnosed at the following ages: 2 years 6 months, 3 years 1 month, 3 years 6 months and 5 years 4 months, due to symptoms associated with pulmonary cystic infection such as fever and cough. If their neonatal condition was stable, surgery was electively planned after the age of one. In addition, C-VATS lobectomy was also considered when differential lung ventilation for thoracoscopic surgery was deemed technically feasible based on the body size of infants. Mean age and weight for surgery was 2 years 3 months (median 21: range $12-68$ months) and $12.2 \mathrm{~kg}$ (median 11.5: range $7.3-17.8 \mathrm{~kg}$ ). The locations of malformation were 3 right lower lobes, 3 left upper lobes, and 3 left lower lobes (Table 1).

\section{Surgical outcome and morbidity}

Pulmonary resection consisted of the following: lobectomy in 8 cases, segmentectomy in 1 case, wedge resection in 2 cases, fractionated lung resection in 1 case and lobectomy 
Table 1 Patient characteristics

\begin{tabular}{|c|c|c|c|c|c|c|c|c|}
\hline Case & Age of surgery & Sex & Height (cm) & Weight (kg) & Diagnosis time & Symptom & Malformation site & Stocker classification \\
\hline 3 & 7 days & M & 50 & 2.6 & Prenatal & Respiratory failure & RUL & Type 3 \\
\hline 5 & $1 Y 0 M$ & $\mathrm{~F}$ & 72 & 7.9 & Prenatal & None & LUL & Type 1 \\
\hline 6 & $1 Y 1 M$ & $\mathrm{~F}$ & 69 & 7.3 & Prenatal & None & RLL & Type 2 \\
\hline 7 & $1 Y 2 M$ & $\mathrm{~F}$ & 76 & 9.8 & Prenatal & None & LLL & Type 1 \\
\hline 8 & $1 Y 3 M$ & $\mathrm{~F}$ & 72 & 9.3 & Prenatal & $\begin{array}{l}\text { None (cyst } \\
\text { expansion) }\end{array}$ & RLL & Type 1 \\
\hline 10 & $2 Y 9 M$ & M & 90 & 16 & $2 Y 6 M$ & Fever & LLL & Type 1 \\
\hline 11 & 3Y3М & $\mathrm{F}$ & 94 & 15 & $3 Y 1 M$ & Fever, cough & LUL & Type 4 \\
\hline 12 & 3Y8M & M & 96 & 14.9 & $3 Y 6 M$ & Fever & LLL & Type 1 \\
\hline 13 & $5 Y 8 M$ & $\mathrm{~F}$ & 105 & 17.8 & $5 Y 4 M$ & Fever & LUL & Type 2 \\
\hline
\end{tabular}

Y, year; M, month; F, female; M, male; RUL, right upper lobe; RML, right middle lobe; RLL, right lower lobe; LLL, left lower lobe; LUL, left upper lobe.

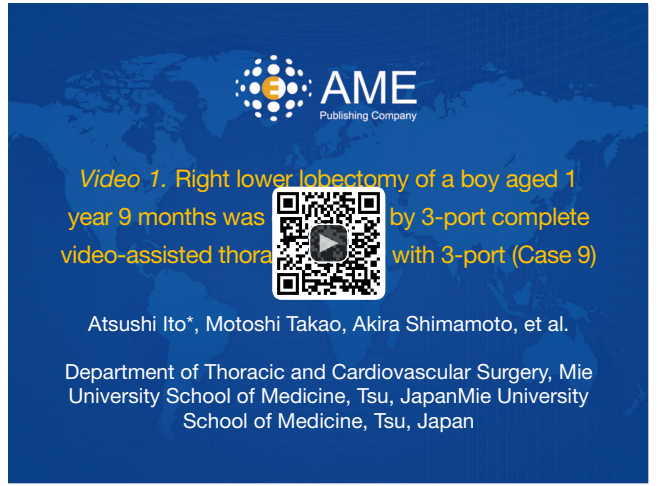

Figure 1 Right lower lobectomy of a boy aged 1 year 9 months was conducted by 3 -port complete video-assisted thoracic surgery with 3-port (Case 9) (5).

Available online: http://www.asvide.com/watch/33038

plus segmentectomy in 1 case. Overall, there were 9 thoracotomy and 4 thoracoscopic surgery. Mean operation time was $162 \mathrm{~min}$ (range, 67-290 $\mathrm{min}$ ) and blood loss was $21 \mathrm{~mL}$ (range, $0-74 \mathrm{~mL}$ ) on average. Pathological examination revealed the following: Type 1 in 6 cases, Type
2 in 4 cases, Type 3 in 1 case, Type 4 in 1 case (one case was indistinguishable). None of the cases showed malignant conversion of the cystic wall. The follow-up period after surgery was $20.5 \pm 17.1$ months. No postoperative complications were observed during hospitalization (Table 2). Moreover, even in the long-term results, all cases survived and there were no complications, such as thoracic deformity or respiratory failure. However, 2 of patients who underwent segmentectomy showed a cystic remnant (Case 4, Case 6).

No statistically significant differences were observed between the thoracotomy Group and C-VATS group in terms of age and height at intervention, operation time, blood loss, postoperative day of drain removal, and length of hospital stay after surgery. However, only the body weight was heavier in C-VATS group $(\mathrm{P}=0.03)$ (Table 3).

At the age of 1 year 9 months (BW $11.5 \mathrm{~kg}$ ), C-VATS lobectomy was successfully performed (Case 9, Figure 1). The CT images and histological features of this case is presented in Figure 2.

Since the surgical instruments for adults cannot be used in infants, we selected the three-part dismantling CLICK line series for pediatric laparoscopic surgery (Karl Stortz, 
Table 2 Surgical outcome

\begin{tabular}{|c|c|c|c|c|c|c|c|c|}
\hline Case & Age of surgery & Approach & Operation & $\begin{array}{l}\text { Operation } \\
\text { time (min) }\end{array}$ & $\begin{array}{l}\text { Bleeding } \\
(\mathrm{mL})\end{array}$ & $\begin{array}{l}\text { Remove drain } \\
\text { (day) }\end{array}$ & $\begin{array}{l}\text { Length of hospital } \\
\text { stay (POD) }\end{array}$ & Complication \\
\hline 1 & 1 day & Thoracotomy & Lobectomy & 109 & 0 & 6 & 16 & None \\
\hline 2 & 4 days & Thoracotomy & Wedge resection & 107 & 30 & 4 & 14 & None \\
\hline 3 & 7 days & Thoracotomy & $\begin{array}{c}\text { Fractionated lung } \\
\text { resection }\end{array}$ & 67 & 2 & $\mathrm{~N} / \mathrm{A}$ & 16 & None \\
\hline 5 & 1YOM & Thoracotomy & Lobectomy & 265 & 74 & 2 & 5 & None \\
\hline 6 & $1 \mathrm{Y} 1 \mathrm{M}$ & Thoracotomy & $\begin{array}{c}\text { Lobectomy + } \\
\text { segmentectomy }\end{array}$ & 180 & 0 & 3 & 5 & Cystic remnant \\
\hline 7 & $1 Y 2 M$ & Thoracotomy & Lobectomy & 155 & 11 & 2 & 8 & None \\
\hline 10 & $2 Y 9 M$ & C-VATS & Lobectomy & 197 & 25 & 2 & 6 & None \\
\hline 11 & 3Y3M & C-VATS & Wedge resection & 78 & 0 & 3 & 9 & None \\
\hline 12 & $3 Y 8 M$ & Thoracotomy & Lobectomy & 167 & 50 & 2 & 7 & None \\
\hline 13 & $5 Y 8 M$ & C-VATS & Lobectomy & 290 & 1 & 3 & 8 & None \\
\hline
\end{tabular}

Y, year; M, month; F, female; M, male; C-VATS, complete video-assisted thoracic surgery; POD, post-operative day.

Table 3 Descriptive statistics of the two groups of infants (Case 5-13)

\begin{tabular}{|c|c|c|c|}
\hline Variable & Thoracotomy $(\mathrm{N}=5)$, median [range] & C-VATS $(\mathrm{N}=4)$, median [range] & $P^{*}$ \\
\hline Height (cm) & 72 [69-96] & 92 [72-105] & 0.11 \\
\hline Weight (kg) & $9.3[7.3-14.9]$ & $15.5[11.5-17.8]$ & 0.03 \\
\hline Operation time (min) & $167[116-265]$ & 188 [78-290] & 0.73 \\
\hline Remove drain (day) & 2 [2-3] & $2.5[2-3]$ & 0.89 \\
\hline Length of hospital stay (day) & $7[5-9]$ & $7[6-9]$ & 0.71 \\
\hline
\end{tabular}

*, $\mathrm{P}$ values refer to the Mann-Whitney $\mathrm{U}$ test. $\mathrm{Y}$, year; $\mathrm{M}$, month; F, female; M, male; C-VATS, complete video assisted thoracic surgery.

Inc., Tuttlingen, Germany) and a $5 \mathrm{~mm}$ rigid endoscope. Intraoperative differential ventilation is also essential for the adequate visualization and exposure of the other lung, especially during the thoracoscopic approach (6). As there is no appropriate size double-lumen tube in neonates and infants, differential lung ventilation with a bronchial blocker is necessary. However, the slip joint between the tracheal tube and the breathing circuit is the narrowest point in the space to maneuver the bronchial fiber and the blocker can be limited especially with the smaller size of tubes $(5.5-\mathrm{mm}$
ID or less) (7). To simultaneously insert the fiber and the blocker into a thin tracheal tube, it is necessary to exchange it with a slip joint of larger size or remove it and insert them separately (Figure 3).

\section{Discussion}

CPAM accounts for $95 \%$ of congenital cystic lung diseases and it is the most common cystic lung lesion diagnosed by prenatal screening at $18-20$ weeks of gestation. A commonly 

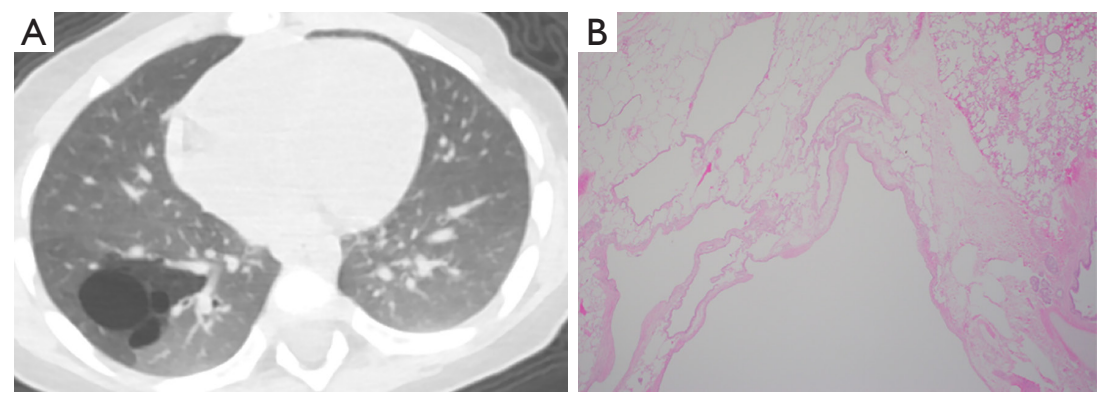

Figure 2 A 1-year-9-month-old boy with type 2 CPAM. (A) Chest CT revealed multi-septated air-filled cystic lesions in the right lower lobe; (B) photomicrograph of type 2 CPAM showed multiple bronchiole-like structures (HE staining, $\times 40$ ). CPAM, congenital pulmonary airway malformation.
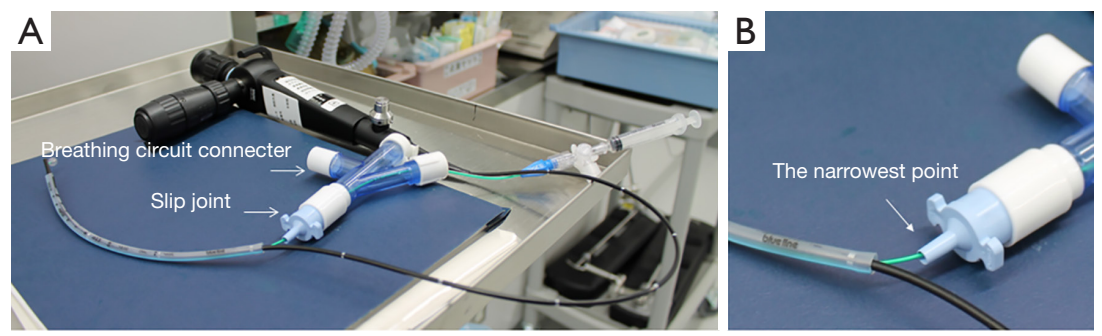

Figure 3 In order to simultaneously insert the bronchial fiber and the bronchial blocker into a thin tracheal tube, it is necessary to remove the slip joint and insert them separately.

quoted incidence of CPAM is 1 per 25,000-35,000 live births (2). CPAMs are usually identified during routine obstetric care due to the advances of ultrasound technology. Additionally, infants with this abnormality most commonly present with respiratory distress and recurrent pulmonary infections (8). Surgical management of asymptomatic CPAM is a highly contested issue in pediatric thoracic surgery. Despite several studies, the timing of surgical treatment and resection procedures has not been clearly defined.

In our cases requiring surgery in the neonatal period, NICU management was necessary due to unstable general and respiratory conditions. Although semi-emergent surgery was required immediately after birth for survival, the prenatal diagnosis of all neonates enabled us to plan the surgical preparation at an early stage. Since differential lung ventilation is difficult in neonates, we performed thoracotomy in all cases. In our cases, there were no serious complications after surgery. This can be explained by the protective effect of the maternal antibody, namely, the high levels of immunoglobulin $\mathrm{G}$ that are transferred to the fetus in utero which are still observed at 1.5 months of age, and decrease in the first 6 months of life $(9,10)$.

Although there seems to be no argument about the surgery in neonates, we should be concerned about several issues with the surgery in infants. The reason why asymptomatic patients with CPAM need surgery in infants is the prevention of infection and malignant conversion of the cystic wall. As in our cases, when their condition is stable without any symptoms or infections, we recommend that they undergo surgery after the age of one for appropriate growth. In addition, we propose that C-VATS lobectomy may be feasible if they are older than 18 months or weigh more than $10 \mathrm{~kg}$ from the viewpoint of vessel/ bronchial dissection technique and differential ventilation during anesthesia (Figure 4).

Wong et al. reported that nearly all of the asymptomatic patients with CPAM at birth developed symptoms by their later pre-school years or even during adolescence (11). However, since the prenatal diagnosis in the report was not advanced as it is today, it is to be noted that the cohort by Wong et al concerns a selected group of patients that cannot be directly compared to patients in current practice. In our study, all cases after 2 years of age underwent surgery 


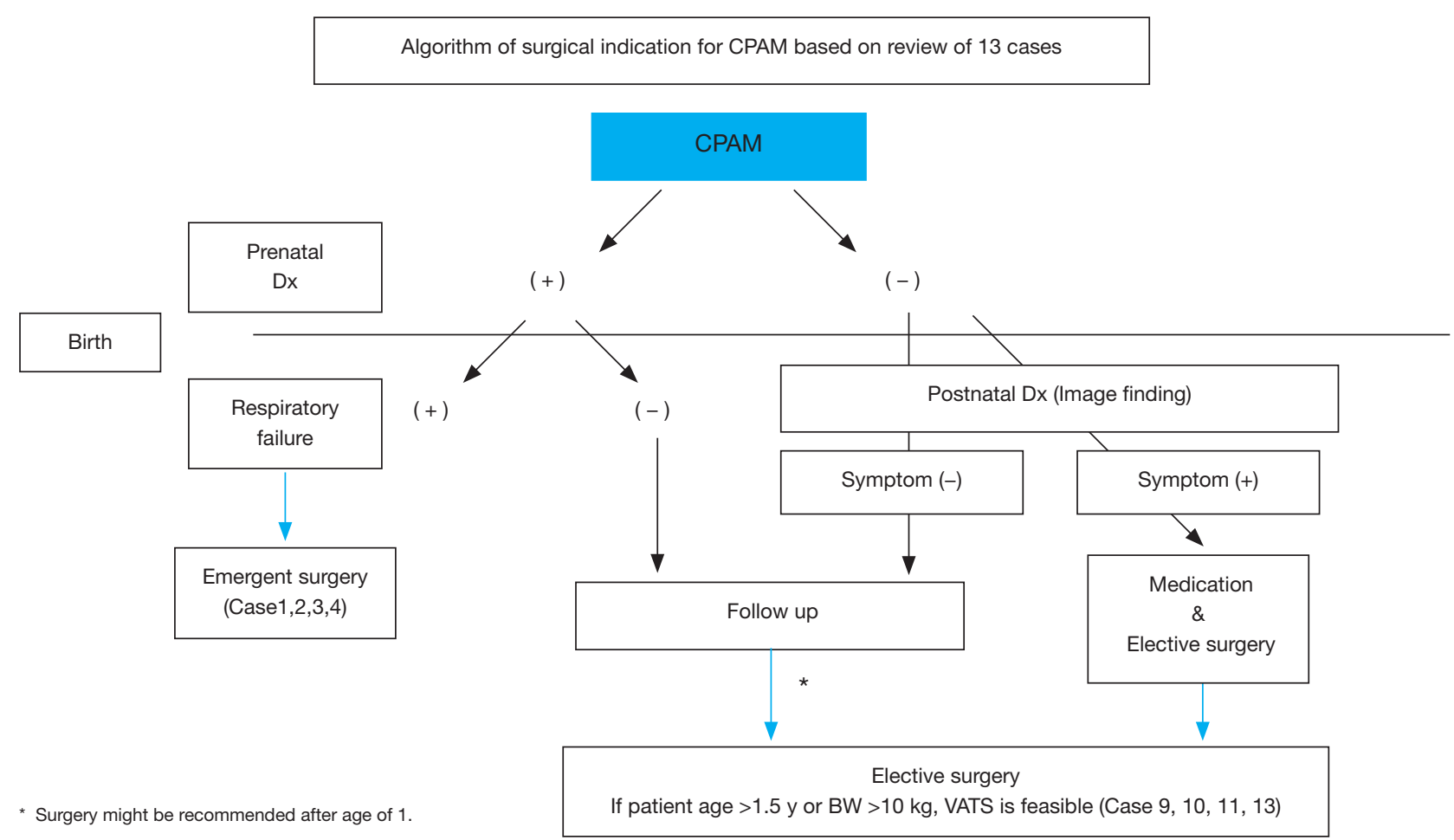

Figure 4 Algorithm of surgical indication for CPAM based on review of 13 cases. *, surgery might be recommended after age of 1 for appropriate growth and safety surgery. CPAM, congenital pulmonary airway malformation; BW, body weight; VATS, video-assisted thoracic surgery.

due to infection. Considering the possible difficulty of surgery in cases of a thoracic adhesion associated with infection, surgery performed between 1 and 2 years might be appropriate in asymptomatic cases.

On the other hand, some reports recommend surgical intervention earlier than our recommendation $(12,13)$. Style $e t a l$. reported that patients who undergo resection at an earlier age (resection $\leq 4$ months) had shorter operative times and duration of anesthesia than the late resection group (resection $>4$ months) (12). However, in their report, two patients with resection at less than 4 months required conversion of thoracoscopic resection to thoracotomy due to bleeding from the pulmonary artery owing to technical issues. Thoracoscopic resection should be avoided if there are technical difficulties since bleeding from the pulmonary artery could be fatal in pediatric surgery. In order to safely perform C-VATS in infants, we recommend waiting to age 18 months or weight of more than $10 \mathrm{~kg}$.

Although the natural course of unresected CPAM is unknown, several studies have shown that some cases will stay asymptomatic for years (14). Furthermore, previous literature reported a paradoxical increase of infections after surgical resection (15). For infants who remain completely asymptomatic, the decision between surgical management and observation is controversial. However, we prefer complete resection of all lesions of CPAM base on reports of the potential for malignant transformation. As no predictive factor of malignant conversion of the cystic wall is currently available, only systematic removal can effectively avoid such conversion. CPAM Type 1 is a precursor of mucinous bronchioloalveolar carcinoma. CPAM Type 4 is a precursor of rhabdomyosarcoma (10). Furthermore, the differential diagnosis between CPAM and pleuropulmonary blastoma is not always easy on imaging. Delayed recognition or failure to resect pleuropulmonary blastoma significantly worsens the prognosis (16). Unlike the method of bullectomy in adult emphysema, it is preferable to resect all lesions of CPAM due to the possibility of malignant transformation of the cystic wall.

Thoracoscopic surgery for asymptomatic patients with CPAM during infancy is now commonly performed, and most reports have high success rates without significant postoperative complications $(17,18)$. In addition, Mattioli et al. noted the superiority of thoracoscopic 
surgery in terms of both the length of postoperative hospitalization compared with thoracotomy and the fact that thoracotomy can induce thoracic deformation and scoliosis (6). CPAM seems to be one of the diseases that has seen the most dramatic increase in application of thoracoscopy (19).

\section{Conclusions}

Patients with respiratory failure after birth due to CPAM require surgery within 1 week, nevertheless, the postoperative course is considerably good. Since early surgical resection of asymptomatic CPAM is often recommended for the prevention of infection and the development of lung malignancy, we recommend performing surgery after the age of 1 year if the patient's condition is stable. Furthermore, C-VATS lobectomy may be feasible if they are older than 18 months or weigh more than $10 \mathrm{~kg}$.

\section{Acknowledgments}

None.

\section{Footnote}

Conflicts of Interest: The authors have no conflicts of interest to declare.

Ethical Statement: The authors are accountable for all aspects of the work in ensuring that questions related to the accuracy or integrity of any part of the work are appropriately investigated and resolved. This study was approved by the institutional ethics board of Mie University Hospital (No. H2019-043). Appropriate written informed consent was obtained for the publication of this report and the accompanying images and videos.

\section{References}

1. Stocker JT. Congenital pulmonary airway malformation: a new name for and an expanded classification of congenital cystic adenomatoid malformation of the lung. Histopathology 2002;41:424-31.

2. McDonough RJ, Niven AS, Havenstrite KA. Congenital pulmonary malformation: a case report and review of the literature. Respir Care 2012;57:302-6.

3. Bolde S, Pudale S, Pandit G, et al. Congenital pulmonary airway malformation: A report of two cases. World J Clin Cases 2015;3:470-3.

4. Aziz D, Langer JC, Tuuha SE, et al. Prenatally diagnosed asymptomatic congenital cystic adenomatoid malformation: to resect or not? J Pediatr Surg 2004;39:329-34.

5. Ito A, Takao M, Shimamoto A, et al. Right lower lobectomy of a boy aged 1 year 9 months was conducted by 3-port complete video-assisted thoracic surgery with 3-port (Case 9). Asvide 2019;6:353. Available online: http://www. asvide.com $/$ watch $/ 33038$

6. Mattioli G, Pio L, Disma NM, et al. Congenital lung malformations: shifting from open to thoracoscopic surgery. Pediatr Neonatol 2016;57:463-6.

7. Disma N, Mameli L, Pini-Prato A, et al. One lung ventilation with Arndt pediatric bronchial blocker for thoracoscopic surgery in children: a unicentric experience. Paediatr Anaesth 2011;21:465-7.

8. David M, Lamas-Pinheiro R, Henriques-Coelho T. Prenatal and postnatal management of congenital pulmonary airway malformation. Neonatology 2016;110:101-15.

9. Lebon A, Verkaik NJ, Labout JA, et al. Natural antibodies against several pneumococcal virulence proteins in children during the pre-pneumococcalvaccine era: The generations $R$ study. Infect Immun 2011;79:1680-7.

10. Fievet L, Natale C, D'Journo XB, et al. Congenital air way malformation and sequestration: Two standpoints for a single condition. J Minim Access Surg 2015;11:129-33.

11. Wong A, Vieten D, Singh S, et al. Long-term outcome of asymptomatic patients with congenital cystic adenomatoid malformation. Pediatr Surg Int 2009;25:479-85.

12. Style CC, Cass DD, Veria MA, et al. Early vs late resection of asymptomatic congenital lung malformations. J Pediatr Surg 2019;54:70-4.

13. Jelin EB, O'Hare EM, Jancelewicz T, et al. Optimal timing for elective resection of asymptomatic congenital pulmonary airway malformations. J Pediatr Surg 2018;53:1001-5.

14. Chetcuti PA, Crabbe DC. CAM: the conservative approach. Arch Dis Child Fetal Neonatal Ed 2006;91:F463-4.

15. Delestrain C, Khen-Dunlop N, Hadchouel A, et al. Respiratory morbidity in infants born with a congenital lung malformation. Pediatrics 2017. doi: 10.1542/ peds.2016-2988. 
16. Delacourt C, Hadchouel A, Khen DN. Shall all congenital cystic lung malformations be removed? The case in favour. Paediatr Respir Rev 2013;14:169-70.

17. Nasr A, Bass J. Thoracoscopic vs open resection of congenital lung lesions: a meta-analysis. J Pediatr Surg 2012;47:857.

18. Kunisaki SM, Powelson IA, Haydar B, et al.

Cite this article as: Ito A, Takao M, Shimamoto A, Kaneda S, Matsushita K, Inoue M, Uchida K. Introduction of thoracoscopic surgery for congenital pulmonary airway malformation in infants: review of 13 consecutive surgical cases. J Thorac Dis 2019;11(12):5079-5086. doi: 10.21037/jtd.2019.12.14
Thoracoscopic vs open lobectomy in infants and young children with congenital lung malformations. J Am Coll Surg 2014;218:261.

19. Rothenberg SS, Kuenzler KA, Middlesworth W, et al. Thoracoscopic lobectomy in infants less than $10 \mathrm{~kg}$ with prenatally diagnosed cystic lung disease. J Laparoendosc Adv Surg Tech A 2011;21:181-4. 